REVISTA DE LA ESCUELA DE CIENCIAS DE LA EDUCACIÓN, AÑO 16, NRO. 15, VOL. 1, ENERO A JUNIO DE 2020. PÁGINAS 21-33. ISSN 2362-3349 (EN LÍNEA). ENTRE EL AFECTO, LA AUTORIDAD Y LA TRANSMISIÓN: ANÁLISIS DE LOS VÍNCULOS INTERGENERACIONALES EN LAS EXPERIENCIAS ESCOLARES DE FAMILIAS MIGRANTES. MARIANA ALEJANDRA GONZÁLEZ

\title{
ENTRE EL AFECTO, LA AUTORIDAD Y LA TRANSMISIÓN: ANÁLISIS DE LOS VÍNCULOS INTERGENERACIONALES EN LAS EXPERIENCIAS ESCOLARES DE FAMILIAS MIGRANTES
}

\author{
Mariana Alejandra González* \\ Universidad de Buenos Aires, Argentina \\ marianaa.gonzalez@yahoo.com
}

Recibido: 24/06/2019 Aceptado: 8/01/2020

\section{Resumen}

Este trabajo se inscribe en el marco de una investigación de maestría, cuyo objeto son las experiencias educativas de adultos/as migrantes y sus hijos/as, que residen en barrios populares de la Ciudad Autónoma de Buenos Aires (CABA). Se hace foco en las relaciones intergeneracionales (padre/madre-hijo/a y docente-estudiante), que tienen lugar en tales experiencias. Para ello se ha realizado un trabajo de investigación cualitativo, basado en entrevistas biográficas y semiestructuradas.

Siguiendo las teorías de la sociología de individuación (Martuccelli, 2006; 2010) y de la experiencia (Dubet 2007; 2013), se procede al análisis del corpus empírico. Se destacan distancias y encuentros en ambas configuraciones vinculares; entre los que sobresale la importancia del afecto, el cual deviene un soporte en las biografías de los actores. Asimismo, se tensionan las configuraciones de autoridad y los modos de participación parentales en la escolaridad de hijos/as.

Palabras clave: Experiencia escolar - Relaciones intergeneracionales - Sociología de la individuación Sociología de la experiencia.

\begin{abstract}
This work is framed in a magister research, which aim was to analyze the scholar experiences of migrants adults and their children, who currently live in popular neighborhood of Buenos Aires City. The focus is on the intergenerational relationships (parents-children and teacher-students), that take place in those experiences. For that it has been done a qualitative research, based on biographical interviews and semi estructured ones.

The analysis proceeds following the theories of sociology of individuation (Martuccelli, 2006; 2010) and sociology of experience (Dubet, 2007; 2010). It is highlighted the distances and meeting between both linking configuration; in which the warmth stands out, as becoming a support in actor's biography. Likewise, it stresses the authority configuration and the way parents participate in the scholarity of their children.
\end{abstract}

Keywords: Scholar Experience - Intergenerational Relationships - Sociology of Individuation - Sociology of Experience.

*Magíster en Ciencias Sociales con Orientación en Educación, por FLACSO Argentina. Licenciada en Psicología, Universidad de Buenos Aires (UBA). Becaria doctoral del Instituto de Investigaciones Gino Germani, Facultad de Ciencias Sociales, UBA. 
REVISTA DE LA ESCUELA DE CIENCIAS DE LA EDUCACIÓN, AÑO 16, NRO. 15, VOL. 1, ENERO A JUNIO DE 2020. PÁGINAS 21-33. ISSN 2362-3349 (EN LÍNEA). ENTRE EL AFECTO, LA AUTORIDAD Y LA TRANSMISIÓN: ANÁLISIS DE LOS VÍNCULOS INTERGENERACIONALES EN LAS EXPERIENCIAS ESCOLARES DE FAMILIAS MIGRANTES. MARIANA ALEJANDRA GONZÁLEZ

\section{Introducción}

La población migrante en Argentina representa el 4,5\% del total, se ubica principalmente en el área Metropolitana de Buenos Aires y realiza trabajos de construcción, tareas domésticas y textiles. Su llegada comienza a ser registrada en tiempos de gestación de la nación, como proveniente de Europa. Desde de 1945 se reconoce la presencia de extranjeros ${ }^{1}$ limítrofes que, ubicados en las fronteras, realizaban tareas agrícolas. En 1960, debido a la crisis del campo, éstos se movilizan hacia Buenos Aires (zona industrial) y se instalan en las villas de la ciudad (Vaccotti, 2014), siendo emplazamientos con carencias materiales y sanitarias (Cravino, 2009). Desde entonces, se construye una imagen del migrante regional estigmatizada. La influencia de los medios de comunicación y las expresiones de políticos, lo posicionan como usurpador, ladrón, oportunista y/o enfermo (Beheran, 2012).

Según el último censo (2010), en las villas de CABA residen 163.587 personas, de las cuales el $34 \%$ proceden de países extranjeros. A su vez, de aquellos jefes de familia de origen regional, el $69 \%$ de sus hijos son nacidos en CABA y el $31 \%$ son extranjeros. Si bien la mayoría de tales niños son argentinos, son tratados estigmatizados y discriminados- como si fueran extranjeros. La peyorativa denominación "migrantes de segunda generación" invisibiliza la subjetividad de cada uno, al quedar subsumidos bajo el rasgo étnico (Juliano, 2002; Novaro, Padawer y Hetch, 2015).

El abordaje de estas complejidades presentes en las experiencias sociales de personas migrantes exige un cambio de mirada sociológica. En la actualidad, cada vez más investigaciones sociales se centran en los individuos, sus experiencias y reflexividades. En esta línea se posiciona la sociología de la individuación de Danilo Martuccelli, la cual se interroga por el tipo de individuo que es estructuralmente fabricado por una sociedad en un período histórico (2006; Martuccelli y De Singly, 2012; Araujo y Martuccelli, 2012). Se vale de dos operadores analíticos, que permiten el estudio de las vinculaciones entre lo social y lo individual: los soportes -capacidades existenciales y sociales del individuo de sostenerse en el mundo- y las pruebas -desafíos históricos, socialmente producidos, culturalmente representados y desigualmente distribuidos comunes a una sociedad por la que atraviesan los sujetos. En el marco de una tesis de maestría (González, 2018), este trabajo tiene por objetivo analizar las relaciones intergeneracionales, entre padres e hijos, que acontecen en las experiencias educativas.

\section{Estrategia metodológica}

Esta investigación es de tipo cualitativo, cuya fortaleza radica en captar fenómenos de causalidad y de relación mejor que los enfoques cuantitativos (Souza, 2009). Desde dicha perspectiva, se procedió con un estudio cuya muestra, de selección intencional, se integra por siete personas adultas migrantes (sin incluir criterio de nacionalidad), que tuvieran niños/as a cargo, que asistieran a escuela pública primaria del barrio Bajo Flores, CABA. El contacto se realizó mediante la recomendación de una informante clave en primera instancia y luego, por bola de nieve. Adicionalmente, a fin de propiciar la polifonía de voces, se ha entrevistado a una docente y una bibliotecaria de la única escuela primaria ubicada en el barrio. Ellas fueron seleccionadas por ser las personas con mayor antigüedad en la escuela y residir en dicho barrio. Las entrevistas semiestructuradas consisten en una conversación registrada y dirigida por un investigador, según un tema de interés, a partir de una línea argumental del entrevistado, a fin de acceder a su interpretación de experiencias, en sus propios términos (Marradi, Archenti y Piovani, 2007).

Por otro lado, con las personas migrantes se han realizado doce entrevistas biográficas, cuyo carácter es más profundo, ya que indaga aspectos significativos personales, en un clima de confianza propicio (Souza, 2009). Se ha seguido el criterio de saturación teórica para determinar la duración y cantidad de entrevistas, por lo que se ha detenido cuando no surgiera información nueva.

En ambos tipos de entrevista -semiestructurada y biográfica-, antes de comenzar, se ha explicado el proyecto de investigación (finalidad, duración, marco institucional, entre otros). Se ha garantizado total confidencialidad, utilizando datos de identificación ficticios en posteriores transcripciones o publicaciones. Todo esto quedó sellado con la firma del consentimiento, por ambas partes.

Siguiendo los lineamientos de la teoría fundamentada (Strauss y Corbin, 2006), se construyeron categorías emergentes en el permanente ida y vuelta entre el trabajo de campo y la lectura del marco teórico. El trabajo de codificación fue realizado con el software Atlas $\mathrm{Ti}$, que facilita la selección de testimonios y su asignación a categorías de análisis.

\footnotetext{
${ }^{1}$ Sin adherir al enfoque de género androcéntrico, pero a fin de facilitar la lectura se utiliza el masculino en las terminaciones plurales.
} 
REVISTA DE LA ESCUELA DE CIENCIAS DE LA EDUCACIÓN, AÑO 16, NRO. 15, VOL. 1, ENERO A JUNIO DE 2020. PÁGINAS 21-33. ISSN 2362-3349 (EN LÍNEA). ENTRE EL AFECTO, LA AUTORIDAD Y LA TRANSMISIÓN: ANÁLISIS DE LOS VÍNCULOS INTERGENERACIONALES EN LAS EXPERIENCIAS ESCOLARES DE FAMILIAS MIGRANTES. MARIANA ALEJANDRA GONZÁLEZ.

Finalmente, el trabajo de campo se desarrolló en la villa 1-11-14, ubicada en el barrio Bajo Flores. Se trata de un emplazamiento comprendido en la zona "C" de la CABA, lo que implica que el $48 \%$ de los hogares no logra cubrir sus necesidades básicas (GCABA, 2012) ${ }^{2}$.

Las villas constituyen barrios con tramas urbanas difusas, disposiciones no amanzanadas, servicios básicos deficientes e irregularidades sobre la tenencia de suelo (Azparren, 2017). Además, se separan de otros barrios por una distancia institucional (Merklen, 2005), al quedar ubicada en una situación de heteronomía respecto

de la ciudad, evoluciona poco y se integra escasamente. En la villa 1-11-14 residen 27.973 habitantes (INDEC, 2010). Un cuarto de esos hogares no disponen de servicio de red cloacal (Suárez, Mitchell y Lépore, 2014) lo que constituye un riesgo sanitario y repercute también en las condiciones ambientales del barrio. Alrededor del $45 \%$ de la población que trabaja, lo hace en el mismo barrio, lo que podría remitir a una menor salida y circulación hacia otras zonas de la ciudad. Se destaca que la población migrante presenta mayores de tasas de actividad laboral que la población nativa.

\section{Vínculos intergeneracionales en experiencias escolares}

En esta sección se desarrollan las características salientes de las interacciones docente-alumno, tanto en las experiencias escolares ${ }^{3}$ de los entrevistados, así como en la de los niños en la actualidad (ambas son recuperadas desde los testimonios de padres y madres). En aquellas, los vínculos estaban mediados por modos disciplinares:

En Bolivia, ponle que... no te exigían que tengas ropa limpia, pero bien remendadito, limpito, las uñas bien cortadas, y no tenías que faltarle el respeto al profesor, tenías que cumplir con... con los deberes de la escuela y sino, le llamaban a tu papá. Era muy estricto. Si te portabas mal, te ponían al chancho, como dicen, a un rincón con los pies para arriba. Y si no te hacían... te pegaban acá [Señala su mano] o te jalaban del pelo o te tiraban de la oreja. Eran muy estrictos. Así no tengas un buen zapato limpio... o sea roto, pero tiene que estar limpito (Ester).

Tal como puede verse, los entrevistados dan cuenta de sus experiencias escolares, desde un ordenamiento institucional tradicional; ubican, en este sentido, a una escuela disciplinar, preceptores inapelables (posiciones asimétricas), padres que acatan la decisión de maestros. A este tipo de escuela se refieren Dubet y Martuccelli (2000), cuando expresan que desde un enfoque moderno clásico se piensa a las instituciones tradicionales (familia, escuela, iglesia) como fundamentales de la sociedad, por cuanto garantizan la estabilidad y reproducción de un orden, al transformar los valores en normas y éstas en personalidades individuales.

A su vez, la escuela y fundamentalmente las relaciones que tienen lugar allí, devienen un soporte en sus biografías. En la medida en que señalan que la contención, la escucha, los tratos amorosos recibidos, constituyen un sostén con el que cuentan. Es interesante observar que la escuela tradicional, aún con su lógica disciplinar y normalizadora, se consolida como una institución de apoyo afectivo. Los docentes, si bien encarnan una posición de autoridad moral, respetable y sin fisuras, logran a la vez ubicarse cercanos a los alumnos, contenedores, amorosos. Se construye una relación pedagógica en una mutua sujeción de amor y deber entre el alumno y el maestro (Rosseau, 1982).

Es importante mencionar que dichos tránsitos escolares tienen lugar en un contexto de vulnerabilidad socioeconómica, donde los participantes cumplían con deberes domésticos, sino laborales y contaban con poco apoyo parental para continuar sus estudios. Allí, la escuela se consolida como un refugio que "(...) ante la escasez de otros amortiguadores materiales y simbólicos, se establecen como soportes de la vida y producen al mismo tiempo transformaciones en sus espacios de sociabilidad" (Sustas y Touris, 2013, p.35).

La contención recibida coadyuva a la ubicación de la escuela como un soporte en la biografía de las/os participantes. Dicho carácter se reactualiza como el elemento más valorado en la escolaridad de los

\footnotetext{
${ }^{2}$ Dirección General de Estadísticas y Censos - Ministerio de Hacienda, Gobierno de la ciudad de Buenos Aires (2012) Coyuntura económica de la Ciudad de Buenos Aires. http://www.buenosaires.gob.ar/areas/hacienda/sis_estadistico/coyuntura_2012_035.pdf

${ }^{3}$ El concepto experiencia escolar permite problematizar los modos en que se articulan los procesos de socialización y de subjetivación (márgenes de autonomía individual para la producción de sentidos) en la institución escolar contemporánea (Dubet y Martuccelli, 2000).
} 
REVISTA DE LA ESCUELA DE CIENCIAS DE LA EDUCACIÓN, AÑO 16, NRO. 15, VOL. 1, ENERO A JUNIO DE 2020. PÁGINAS 21-33. ISSN 2362-3349 (EN LÍNEA). ENTRE EL AFECTO, LA AUTORIDAD Y LA TRANSMISIÓN: ANÁLISIS DE LOS VÍNCULOS INTERGENERACIONALES EN LAS EXPERIENCIAS ESCOLARES DE FAMILIAS MIGRANTES. MARIANA ALEJANDRA GONZÁLEZ

hijos, dado que destacan la importancia del trato respetuoso y afectivo. Dicen "Se siente muy bien con las seños, le cuidan mucho", "A él le gusta porque dice que la psico operadora que está ahí, le ayuda mucha, cada vez que él está sentado en la escuela, le ve que está ahí se acerca, le pone a leer juntos". La contención y la escucha han sido, y son, componentes muy potentes, de manera tal que hoy, quince años más tarde (como mínimo), los consideran como lo más importante de la escolaridad de sus hijos. Su potencia no solo radica en la permanencia en el tiempo, vía recuerdo hoy reivindicado, sino en las marcas que dejó en su subjetividad.

Tales vivencias remiten a la igualdad individual de oportunidades, que refiere al reconocimiento singular de cada uno. En un contexto donde los actores, siendo niños, debían cumplir con muchas obligaciones y vivían en situaciones de vulneración, encontraban en la escuela reconocimiento. Las maestras han ocupado el lugar de otro significativo, tal como lo entiende François De Singly, puesto que brindan validación personal por los logros alcanzados, más allá de la objetivación del mundo (Martuccelli y De Singly, 2012). Entonces, el haberse sentido reconocidos de ese modo, puede haber dejado una marca muy profunda en su subjetividad que se expresa hoy en la valoración del aspecto contención, en la escolaridad de los niños.

Por lo tanto, los vínculos intergeneracionales (docentes-estudiantes) en ambas experiencias escolares se caracterizan por ser afectuosos. En la escolaridad de los padres, estos tratos tenían lugar a la par que prácticas disciplinares y normativas. En la escolaridad de los niños, como se detalla en las siguientes secciones, hay una carencia de tales prácticas, aunque persisten los tratos amorosos.

\section{Ejercicio de autoridad en las experiencias escolares}

Durante las experiencias escolares de los entrevistados, las/os docentes, en tanto representantes de una institución moderna estatal, se presentan como inapelables frente a los alumnos y los padres. Por consiguiente, los vínculos intergeneracionales se configuran en torno a la obediencia.

Lo que noto mucho es la educación, es decir, muy el respeto por el otro, la educación hacia los docentes, este, y los padres también, muy marcado el respeto hacia el docente. Como que el docente viene a ser, no sé, como tu segundo padre, respetás a tu padre, respetás al docente lo que te diga. Si bien estarás de acuerdo o no, pero te toca escucharlo. Siempre fue eso (...) (Dominique).

Max Weber (1983) versa acerca de la legitimidad de la autoridad, en la propia voluntad de ser obedecida; introduce tres tipos: tradicional, racional y carismática. El primer tipo -tradicional- es aquella que se sustenta en la tradición y la costumbre. Una autoridad racionalburocrática es la que depende de los roles de los sujetos que integran una misma institución. Tanto la forma tradicional como la racional no son personales de alguien, sino que se deben al lugar que éste ocupa. Por lo tanto, otra persona en una posición idéntica también será reconocida como autoridad. En tercer lugar, la carismática es aquella que se basa en los atributos del líder. Esta forma no puede ser delegada ni tampoco transferida, solo le corresponde a tal sujeto y mientras conserve esos atributos. En un contexto actual este tipo de autoridad, siendo el más frágil, es el único que subsiste.

Según los entrevistados, se observa que el tipo de autoridad que tiene lugar en sus experiencias escolares es de tipo tradicional y racional burocrático. De modo similar se expresa Paola Gallo (2009) quien analiza los testimonios que remiten al reconocimiento de la autoridad entre las décadas 1940 y 1960 , basados en nociones de disciplina y respeto. Se trata de maneras tradicionales del ejercicio de autoridad, que incluyen castigos físicos, tirar del pelo o de la oreja. Además, ubica otras maneras, como son el reto o la penitencia, propias de instituciones modernas, tales como la escuela. En la familia ocurren castigos similares, que constituyen una base para los ejercicios de autoridad, tradicionales y modernos, en la escuela. Se trata de una continuidad, donde el ejercicio parental constituye una fuente de legitimidad para los/as docentes (Gallo, 2009).

El orden escolar se funda a partir de una relación de subordinación. Los adultos despliegan autoridad sobre los niños. Aquí radica el sentido básico de la educación, tal como la define Emile Durkheim (1984) en relación con la acción que ejercen los adultos sobre los niños, con el fin de producir estados físicos, intelectuales y morales, acordes a la sociedad. En este sentido, el testimonio de Dominique refleja que la autoridad parental está en consonancia con la escolar. Ambas son de tipo tradicional, puesto que el respeto es producto de una posición que así lo demanda (Weber 1983). importantes:

Ahora bien, en un tiempo presente, en la escolaridad de los niños, el ejercicio de autoridad sufre variaciones

E: Eh, también cambiaron muchas cosas de esos años a acá. Porque antes tenía más autoridad el maestro y ahora no. Es más si alguna pequeña cosa el maestro dice en falso, algo, viene la mamá y le va a cagar a trompadas. (...) Eso allá en Bolivia no pasaba, todo lo contrario. Si alguna vez mi papá iba al colegio, 
REVISTA DE LA ESCUELA DE CIENCIAS DE LA EDUCACIÓN, AÑO 16, NRO. 15, VOL. 1, ENERO A JUNIO DE 2020. PÁGINAS 21-33. ISSN 2362-3349 (EN LÍNEA). ENTRE EL AFECTO, LA AUTORIDAD Y LA TRANSMISIÓN: ANÁLISIS DE LOS VÍNCULOS INTERGENERACIONALES EN LAS EXPERIENCIAS ESCOLARES DE FAMILIAS MIGRANTES. MARIANA ALEJANDRA GONZÁLEZ

porque le mandaban una nota o algo, mi papá le autorizaba al maestro que "si mi hija se porta mal castíguele"

M: Y vos decís que eso hoy acá no pasa

E: Acá no pasa.

M: Todo lo contrario, si la madre se tiene que pelear con la maestra, se pelea... (Ester).

Gallo (2009) sostiene que desde 1960-1980 en adelante se produce una transformación en las relaciones asimétricas, más precisamente un achicamiento de las distancias. Con el paso del tiempo, el respeto surge a partir de la relación entre docente y alumno, "(...) ya no todos los adultos de la institución son portadores de autoridad -0 al menos de una autoridad reconocida- sino sólo aquellos que tienen 'personalidad' o que cumplen con determinados requisitos. Consecuentemente la misma ya no es transferible" (Gallo, 2009, p.17). La autora también incluye la dimensión temporal, puesto que en un pasado tales prácticas (ejercicio de autoridad vertical) estaban naturalizadas y hoy se las califica de modo negativo. Esto conlleva a una actitud de control de padres hacia docentes, como se consigna a continuación.

Por lo tanto, siguiendo los modelos de Weber y el estudio de Gallo, se observa que durante la escolaridad de los entrevistados, el maestro se posiciona desde una autoridad racional-burocrática y tradicional, por lo que es obedecido indiscutidamente. Con el paso del tiempo y el cambio de contexto, las familias se encuentran con el tipo carismático de autoridad, que se sostiene a partir de la personalidad. Es posible considerar que los padres atentos a la fragilidad de dicha posición, ahora la demandan y controlan.

\section{"Yo estoy todos los días en la puerta del colegio". Cambios en las configuraciones institucionales y efectos en sus relaciones}

Los puntos anteriores recuperados en la Tabla 1, permiten reflexionar acerca de transformaciones que sufren las instituciones y que tensionan los procesos subjetivos.

Tabla 1. Experiencias escolares de padres e hijos, según configuración de los vínculos intergeneracionales y ejercicio de autoridad

\begin{tabular}{|c|c|c|}
\hline 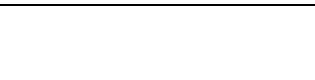 & $\begin{array}{l}\text { EXPERIENCIA ESCOLAR DE LOS } \\
\text { PADRES }\end{array}$ & EXPERIENCIA ESCOLAR DE LOS HIJOS \\
\hline \multirow{2}{*}{$\begin{array}{l}\text { Vínculos } \\
\text { intergeneracionales }\end{array}$} & $\begin{array}{l}\text { Trato afectuoso. Escuela como refugio y } \\
\text { soporte. }\end{array}$ & $\begin{array}{l}\text { Trato afectuoso, contención, escucha como } \\
\text { lo central. }\end{array}$ \\
\hline & $\begin{array}{l}\text { Prácticas disciplinares en tensión con la } \\
\text { contención brindada. }\end{array}$ & $\begin{array}{c}\text { No hay prácticas normativas. Esto genera } \\
\text { críticas y quejas. }\end{array}$ \\
\hline \multirow{3}{*}{$\begin{array}{l}\text { Ejercicio de } \\
\text { autoridad }\end{array}$} & $\begin{array}{c}\text { Autoridad docente inapelable. Incluye la } \\
\text { presencia de castigos físicos, penitencias y } \\
\text { retos. }\end{array}$ & $\begin{array}{l}\text { Autoridad del docente no está más } \\
\text { garantizada. La misma se reconstruye en } \\
\text { cada relación. }\end{array}$ \\
\hline & $\begin{array}{l}\text { Hay continuidad entre ejercicio de } \\
\text { autoridad en la familia y en la escuela. }\end{array}$ & $\begin{array}{l}\text { No hay más obediencia garantizada. } \\
\text { Ruptura de alianza familia-escuela. }\end{array}$ \\
\hline & Tipos tradicional y racional burocrático. & Tipo carismático. \\
\hline
\end{tabular}

Fuente: Dato de trabajo de campo.

Tal como lo detallan los estudios sociológicos actuales, las instituciones modernas están sufriendo mutaciones en cuanto a sus formatos, sentidos, funciones.

La mayoría de los manuales de sociología afirman sin ninguna vacilación que la familia, la escuela, la iglesia, son instituciones fundamentales de la sociedad, que aseguran su reproducción y su estabilidad, que producen actores adaptados a las necesidades de la sociedad. Esta representación ya no corresponde a la vida social contemporánea o a la manera como se la lee. Ninguna de estas instituciones funciona según el modelo clásico, como aparatos capaces de transformar los valores en normas, y las normas en personalidades individuales. No se trata solamente de la "crisis" de las instituciones, de una simple prueba de adaptación a nuevas condiciones. La desintitucionalización señala un movimiento más profundo, una manera totalmente distinta de considerar las relaciones entre normas, valores e individuos, es decir un modo absolutamente distinto de concebir la socialización (Dubet y Martuccelli, 2000, p.201). 
REVISTA DE LA ESCUELA DE CIENCIAS DE LA EDUCACIÓN, AÑO 16, NRO. 15, VOL. 1, ENERO A JUNIO DE 2020. PÁGINAS 21-33. ISSN 2362-3349 (EN LÍNEA). ENTRE EL AFECTO, LA AUTORIDAD Y LA TRANSMISIÓN: ANÁLISIS DE LOS VÍNCULOS INTERGENERACIONALES EN LAS EXPERIENCIAS ESCOLARES DE FAMILIAS MIGRANTES. MARIANA ALEJANDRA GONZÁLEZ.

En lo que sigue, se recorre el texto "El declive y las mutaciones de la institución" (Dubet, 2007) para dar cuenta de este cambio de contexto, que evidencia el relato de las experiencias escolares de los padres, allá, y de los niños, acá.

En primer lugar, Dubet (2007) sostiene que la crisis de las instituciones se inscribe en la mutación del proceso de trabajo sobre el otro. Esto incluye la socialización, el modo de instituir actores, la formación de individuos. En su texto señala las principales características del programa institucional que ordena las lógicas modernas, hasta llegar a nuestros días, donde su retroceso da lugar a un rol activo de los individuos en su deber de justificación propia. En el caso de la escuela, en un tiempo pasado, utiliza técnicas para transmitir valores sagrados, situados por encima de la sociedad. El maestro es obedecido indiscutidamente, sin exponerse, ya que era preservado por la institución y su rol. Las conductas de los alumnos son previsibles. Las personalidades (del maestro y del alumno) se ocultan tras los roles. Esta misma escuela tradicional que describe Dubet (2007) es la de los migrantes entrevistados, en sus trayectorias escolares.

A continuación, el autor desarrolla el declive del programa institucional: "El capitalismo y su crisis, los medios de comunicación, la pobreza y el paro, y la crisis de la familia, han acabado por romper la alianza entre la escuela y la sociedad" (Dubet, 2007, p.14). Los individuos no se identifican más totalmente con los principios sagrados, homogéneos y trascendentes; ahora exigen ser reconocidos en sus singularidades. Esta demanda ingresa a la escuela con la masificación escolar, por cuanto aquella debe ser más democrática, hay presión por la igualdad, así como un aumento de la lógica competitiva. La escuela debe formar en aquellas habilidades requeridas por el mercado. Los maestros, ahora cuestionados, han de demostrar sus competencias para el ejercicio de la profesión.

Concluye el autor, "El declive del programa institucional no significa la muerte de las instituciones, sino la transformación de las instituciones enfrentadas a un mundo más moderno, más desencantado, más democrático y más individualista" (Dubet, 2007, p.28). Justamente, la escuela no dejó de ser fundamental para los entrevistados, sino que ahora esa importancia reposa sobre otras demandas, cobrando importancia la utilidad de los aprendizajes.

Esta nueva configuración de las instituciones se ve reflejada en las experiencias escolares de los niños, particularmente en la vinculación familia-escuela. Se observa que los mandatos escolares no son más reconocidos como legítimos y obedecidos directamente. Los entrevistados manifiestan una gran preocupación por el desarrollo escolar de los hijos, lo que se traduce en diversas prácticas: ayuda en los deberes, acompañar en salidas escolares, asistir a reuniones de padres, apariciones espontáneas en la escuela para charlar con la maestra, diálogos con otros padres, elección de una escuela (y no otra), etc.

M: Respecto del colegio de Joel, vos me decías que vos cuando necesitás algo, vas a la escuela, hablás con la seño, ella te explica y demás.

E: Sí, sí.

$\mathrm{M}$ : ¿Eso sucede seguido, de ir a la escuela de Joel?

$\mathrm{E}$ : Yo estoy todos los días en la puerta del colegio, si tengo alguna duda o algo, me acerco y le pregunto a la maestra. Y siempre estoy...

M: ¿Siempre a la salida?

E: Siempre a la salida o a la entrada, ponle, veo que está ahí, cerquita mío, bueno pregunto cómo está Joel, se porta bien... (...)

$\mathrm{M}:$ ¿Te explicó todo bien?

E: Sí, me explicó, hoy también estuve hablando un ratito con ella.

M: Y siempre que hablás con la señorita es yendo vos a la escuela, no es con una nota, por ejemplo con una cita.

E: No, no, nunca me manda una nota. Ponle que Joel por ahí se portó mal o algo, o me manda una nota para que quiere hablar conmigo, no. Siempre es para preguntarle cómo es, o tengo alguna duda sobre qué pruebas tiene que tener (Ester).

Todos los participantes han coincidido en este punto: hay una preocupación por la escolaridad de los hijos, que se evidencia en prácticas concretas. Ellas representan modos de ejercer control sobre la tarea docente y el desempeño escolar del niño. En este sentido, Kathya Araujo (2016) introduce argumentos para reflexionar en torno al ejercicio de autoridad, desde los cuales puede comprenderse el proceso de mutación institucional y su incidencia en la relación familia-escuela.

En primer lugar, la autora observa que en Chile no siempre hay desobediencia abierta, sino más bien procesos subrepticios de cuestionamiento de la autoridad, como son las quejas, cumplir de mala gana, la burla, etc. En dichos casos, la obediencia es consentida pero no conciliada. A los individuos no les gusta identificarse como sometidos, esto repercute en su autoestima (Araujo, 2016). En los dichos de los entrevistados se refleja tal actitud puesto que, si bien en un primer momento critican o cuestionan a las maestras, finalmente sí acatan lo que ellas dicen. El hecho de cuestionarlas, los aleja de la posición de sumisos. 
REVISTA DE LA ESCUELA DE CIENCIAS DE LA EDUCACIÓN, AÑO 16, NRO. 15, VOL. 1, ENERO A JUNIO DE 2020. PÁGINAS 21-33. ISSN 2362-3349 (EN LÍNEA). ENTRE EL AFECTO, LA AUTORIDAD Y LA TRANSMISIÓN: ANÁLISIS DE LOS VÍNCULOS INTERGENERACIONALES EN LAS EXPERIENCIAS ESCOLARES DE FAMILIAS MIGRANTES. MARIANA ALEJANDRA GONZÁLEZ

A su vez, Araujo (2016) aborda la relación entre disciplina y autoridad en actuales sociedades latinoamericanas. Los sujetos consideran que una autoridad debe ser fuerte para asegurar su obediencia, cercana a un estilo autoritario. "La percepción generalizada no es que la autoridad pueda ser ejercida de forma autoritaria, sino que todo ejercicio de la misma es preconcebido como autoritarismo" (Araujo, 2016, p.68). En este sentido, los entrevistados, quienes habiendo asistido a escuelas donde la figura del maestro era inapelable y se le debía obediencia, hoy transfieren dicha imagen, ahora anhelada, a los docentes de sus hijos. Es decir, la maestra con autoridad que ellos tuvieron en sus primarias, persiste cual ideal para las trayectorias de sus niños a cargo.

En esta nostalgia, vuelta reclamo por lo que ellos experimentaron durante su escolaridad, que consideran una virtud, pero que hoy sus hijos no tienen, se expresan los mandatos tradicionales de la institución moderna. La mutación de la escuela se explicita en la queja de los entrevistados. En su ideal de escuela continúan vivos los formatos escolares tradicionales. Esto produce una distancia entre lo que desean y lo que encuentran.

Araujo (2016) considera que se produce una tensión central respecto del ejercicio de la autoridad en diversos ámbitos sociales. Por un lado, se repudian las prácticas autoritarias. Sin embargo, a la hora de dirigirse hacia el otro, en busca de obediencia, las consideran las más eficientes, lo que hace que sean reproducidas. Esta tensión tiene lugar en un contexto de mayor demanda hacia vínculos horizontales y democráticos, de posicionamiento de los actores a partir de habilidades personales, relaciones con los otros y distanciamiento (hasta desconfianza) respecto de las instituciones. El motivo por el cual los actores reproducen prácticas autoritarias se debe al temor a los subordinados, no solo que no obedezcan, sino también que no puedan ser controlados.

En los testimonios de los participantes tiene lugar dicha tensión. Por un lado, los migrantes anhelan las prácticas disciplinares de sus escuelas primarias, donde las maestras tenían autoridad indiscutida. Sin embargo, dicha exigencia entra en disputa con el horizonte de vínculos horizontales, mayor participación en la escolaridad de los hijos y diálogo con las docentes, desde los que construir una alianza familia-escuela. También es opuesta a los tratos afectuosos y de contención que son los más destacados en las experiencias escolares de los hijos.

A su vez, la supervisión que padres y madres ejercen sobre docentes, puede remitir a una desconfianza, así como una fractura de aquel lugar indiscutido del líder (tradicional/racional), siendo que hoy se posicionan más desde un tipo carismático. Dicha supervisión puede ser vista como una obediencia de modo subrepticio, es decir no conciliada.

\section{"Les digo que no sean como nosotros". Otros sentidos en torno a la supervisión de la escolaridad}

La sección anterior señala aspectos institucionales y contextuales de la vinculación intergeneracional, puntualmente en el ejercicio de autoridad, a la vez que enmarca la relación de los padres con docentes, haciendo foco en el control que ejercen, por medio de diversas prácticas. Ahora bien, en estos modos de controlar a las maestras y al desempeño escolar de los hijos, también se expresan otros sentidos.

En primer lugar, Araujo sostiene que "(...) los padres son medidos y se miden a sí mismos en cuanto individuos, en una proporción altamente significativa, tomando como referencia el destino de los hijos" (Araujo, 2016, p.100). Es decir, las personas adultas estiman su valoración según el logro social de los hijos (sus estudios, familias, trabajos, movilidad social), en ello redunda la importancia de gestionar adecuadamente la autoridad. Por lo tanto, detrás de la actitud de los entrevistados, de supervisar a las maestras, cuestionarlas, así como también en el apoyo activo hacia los hijos, se estaría jugando su propia estima. En la medida en que el destino de los hijos condiciona la valoración de los padres, resulta esperable que éstos dediquen tantos esfuerzos en garantizar un buen desenlace.

En segundo lugar, la escuela es importante porque puede garantizar progreso social. Por ello, a fin de asegurar un tránsito satisfactorio debe ser fiscalizado. Mario refiere en torno al abandono escolar de su hijo mayor:

M: Le digo "Lo primero que tienes que hacer es estudiar", le he dicho. Yo siempre le digo eso. Y les digo, digamos, de que no sean como nosotros

$\mathrm{E}:$ ¿En qué sentido?

M: De estar así, trabajando, en la costura, hay veces como le digo, cuando yo llegué trabajaba en la costura, entraba a las 6 de la mañana, descansaba a la 1, 2, 3 de la madrugada. O sea que no descansaba. Es por eso que les digo "No sean así. Estudien para ser bachilleres. Tener una profesión. Y ahí si quieres vas a ir a trabajar, sino no. Pero ya vas a tener una profesión, donde estar, eh, más acomodado, inclusive ir a trabajar", "Bueno, bueno" me dicen (Mario).

Puede verse que para el entrevistado la educación brinda la posibilidad de lograr una movilidad social ascendente, presume que tener un título secundario habilita a su hijo a realizar otro tipo de trabajos, más calificados en comparación con aquel que él mismo desempeña.

Araujo y Martuccelli (2012), a partir de su trabajo con población chilena, sostienen que la educación se presenta como una promesa individual de movilidad social. 
REVISTA DE LA ESCUELA DE CIENCIAS DE LA EDUCACIÓN, AÑO 16, NRO. 15, VOL. 1, ENERO A JUNIO DE 2020. PÁGINAS 21-33. ISSN 2362-3349 (EN LÍNEA). ENTRE EL AFECTO, LA AUTORIDAD Y LA TRANSMISIÓN: ANÁLISIS DE LOS VÍNCULOS INTERGENERACIONALES EN LAS EXPERIENCIAS ESCOLARES DE FAMILIAS MIGRANTES. MARIANA ALEJANDRA GONZÁLEZ.

Entre los mayores, entre los que la formación educativa se desarrolló entre mediados de los sesenta y los setenta, el avance en el sistema educativo era indisociable a la vez de una singularidad (ser buen alumno) y de una garantía (su obtención aseguraba el ingreso en las capas medias). Para ellos, y leyendo el proceso actual de su propia experiencia, sus hijos se encuentran en un indudable proceso de movilidad escolar ascendente (Araujo y Martuccelli, 2012, p.86).

Los autores introducen dos aristas: la primera es que se constata una movilidad escolar ascendente, lo que implica que las nuevas generaciones están alcanzando mayores niveles de estudio que sus antecesores. En segundo lugar, que, en aquellos años, atravesar con éxito tal prueba escolar era una garantía para un ascenso social, lo que implica pasar de ocupar lugares inferiores a capas medias de la sociedad.

Por lo tanto, los participantes consideran que la educación es un medio para lograr un ascenso social y que así el futuro de los hijos no sea igual al de ellos. En este sentido, el vínculo intergeneracional se motiva por esta movilidad social para las futuras generaciones, lo que demanda prácticas de control hacia las docentes, hacia los hijos, así como los consejos y charlas a fin de convencerlos en la continuidad escolar.

\section{Transmitir la experiencia, para "que no repitan la historia"}

Los padres transmiten a sus hijos la propia experiencia, por medio de una narración. Así lo muestra el testimonio de Mario quien le cuenta a su hijo la experiencia de su propio abandono escolar y espera con ello convencerlo de que él ahora no deje las clases.

Otra participante ha relatado:

E: El año pasado especialmente, estaba muy rebelde mi hija mayor. Y nosotros hablándole "Hija no hagas esto, seguí estudiando, ¿quieres que te ayude, quieres que te busque un apoyo, un maestro, algo?" Tratando de buscar la forma de que ella no lo deje, porque yo sé lo que es... mi marido le dice, una vez se puso a llorar, le contó: "Mirá hija, yo por no escucharlo a mi padre...", una sola vez su papá se había sentado a hablarle y decirle que siga estudiando, que lo iba apoyar en sus estudios, pero él, en la edad de la rebeldía a los 15 años, los amigos le llevaron por el mal camino. Entonces él le habla, le habla, no se cansa de hablarle a su hija y le dice: "No, no quieras que seas lo que yo fui".

M: (...) si tu familia nunca te apoyaba, ¿de dónde crees que salió este interés por la educación, que tienen ustedes dos hacia sus hijos?

E: Por lo que nosotros pasamos.

M: Es decir por lo que ustedes no pudieron tener o no pudieron recibir

E: Sí. Porque nosotros no tuvimos ese apoyo de mi papá. Nunca, te digo, que nunca se sentaba y nos ha dicho qué queremos estudiar, si queremos seguir, si él nos apoyaba, nos ayudaba con los materiales y los útiles (Ester).

Richard les dice a sus hijos que aprovechen las oportunidades: "Yo no quiero que desperdicien, no quiero que cometan los mismos errores, no cometas tomar la misma decisión" en referencia al propio abandono escolar. Él, junto con el testimonio de Ester y Mario, permite reflexionar en torno a la relación intergeneracional.

En las experiencias escolares de los entrevistados, ellos no contaban con un apoyo activo por parte de sus padres, ya que no eran estimulados a continuar con sus estudios, más bien debían cumplir con tareas domésticas o laborales. En un momento presente, en cambio, se comprometen con la escolaridad de los niños a cargo. Por lo tanto, el modo de ser padres no se corresponde con el que recibieron cuando fueron niños. Se trata de una lógica de subjetivación (Martuccelli, 2010), puesto que implica la capacidad de convicción, crítica y acción autónoma, tomando distancia del orden de las cosas.

En las relaciones de padre-hijo se produce una transmisión, que implica un pasaje, de conocimiento, modos de hacer, culturas, tradiciones, historias (Nicastro y Greco, 2009). Los receptores no se desempeñan de manera pasiva, sino que modifican ese legado, de modo que lo puedan portar. El camino de los sucesores no es igual al de los antecesores. La transmisión se opone a la conservación; más bien, impone hacer algo distinto con aquello disponible; se ubica cercana a la creación (Nicastro y Greco, 2009). En este sentido, la apuesta de los entrevistados es que sus hijos no repitan su historia, "que no sean como nosotros", sino que tomándola puedan hacer algo diferente.

Andrés Pedreño (2013) desde un estudio con migrantes marroquíes y ecuatorianos en España, muestra cómo los padres les narran sus experiencias a sus hijos, tratando de incentivar otras opciones, que permitan evitar que la "historia se repita". La educación sería un modo por el que las siguientes generaciones logren "(...) la liberación del estigma de 'inmigrante' que, en el mejor de los casos, les permitiría socialmente 'ser alguien' o por lo menos desarrollar actividades alternativas a las del campo" (Jarrín, 2014, p.5). Los inmigrantes buscan incentivar a sus hijos, mediante su relato, pasar del "logro de no ser nada a ser algo" (Pedreño, 2013, p.69). 
REVISTA DE LA ESCUELA DE CIENCIAS DE LA EDUCACIÓN, AÑO 16, NRO. 15, VOL. 1, ENERO A JUNIO DE 2020. PÁGINAS 21-33. ISSN 2362-3349 (EN LÍNEA). ENTRE EL AFECTO, LA AUTORIDAD Y LA TRANSMISIÓN: ANÁLISIS DE LOS VÍNCULOS INTERGENERACIONALES EN LAS EXPERIENCIAS ESCOLARES DE FAMILIAS MIGRANTES. MARIANA ALEJANDRA GONZÁLEZ.

En esta sección el vínculo intergeneracional está mediado por la transmisión de una experiencia. Los padres narran a sus hijos las propias vivencias de modo tal que no repitan sus errores y que puedan progresar en la carrera escolar.

\section{"Le ayudo en todo lo que puedo". Pasaje del mérito individual al esfuerzo familiar}

Las secciones introducidas previamente decantan en un argumento nodal: antes el éxito escolar era producto del mérito de cada sujeto, hoy, en cambio, es producto del esfuerzo familiar.

Los padres transmiten el legado por medio del relato de su experiencia y con su apoyo activo en la escolaridad (presencia en la escuela, interés y estímulo y ayuda en tareas). Aquí hay una diferencia sustancial con las propias experiencias escolares de los entrevistados, en las cuales, al no contar con el apoyo de sus progenitores, el éxito escolar era producto del propio mérito.

Los entrevistados han dejado la escuela por motivos domésticos (sean situaciones sufridas de violencia o ayudar en quehaceres) o por causas laborales. Ellos no se atribuyen el abandono a razones de poca capacidad intelectual o poco esfuerzo, pero tampoco hacen una lectura amplia de un contexto de vulnerabilidad, en donde la prueba escolar estaba a la par que estas otras tareas; es decir, no destacan causas sociales, estructurales o contextuales. Richard sostiene que dejó el secundario por juntarse con mala amistad, "Malos muchachos que no les importaba el estudio, era boludear. (...) Y yo perdí esa oportunidad".

En este punto, Martuccelli (2006) diferencia entre responsabilidad y responsabilización. La primera remite al hecho de ser responsable por lo que uno hace. La segunda, a la imposición de hacerse responsable por todo lo que le sucede; consiste en:

Hacer que el individuo, cada individuo, tenga que asumir en primera persona el fruto de los fenómenos sociales, que en mucho se explica por factores de interdependencia y ampliamente ajenos a su voluntad. (...) Las instituciones nos imponen cada vez menos modelos, pero nos responsabilizan cada vez más a nivel de nuestras trayectorias (Martuccelli, 2006, p.51).

Se deduce entonces que los entrevistados son responsabilizados por aquello que les sucede, vía el mérito, donde el causal de su fracaso escolar se debe al mal desempeño personal, en dicha prueba.

Ahora bien, en la experiencia escolar de los niños, sobresale que a partir de lo que ellos (padres) vivieron y del valor central que conceden a la escuela, el mérito individual pasa a ser esfuerzo familiar. Es decir, los progenitores se comprometen con la trayectoria escolar de sus hijos, están muy presentes frente a las docentes, así como en sus casas asistiendo con los deberes. La relación intergeneracional está mediada, en parte, por el esfuerzo.

Araujo y Martuccelli (2012) caracterizan a los actores latinoamericanos como Hiper Actores Relacionales, a partir de algunas características centrales, entre las que se destacan las relaciones interpersonales y el esfuerzo. Ambas pueden verse entrelazadas en estos vínculos intergeneracionales y la centralidad de la escolaridad de los niños. Allí se plasma el apoyo recibido por otros, en este caso los padres, y el esfuerzo, entendido como una capacidad de alcanzar metas propuestas, a partir de propios medios, ingenio, arreglárselas.

Una parte importante del esfuerzo que realizan las familias consiste en seguir viviendo en Buenos Aires.

E: No, yo sí, siempre anhelo volver allá, estar allá. Lo que pasa es que hay una... no es lo mismo. Bolivia es un país más pequeño y no hay tantas oportunidades como acá para los jóvenes, ponle, para mis hijos, porque para estudiar una carrera o algo, acá hay muchas ramas de lo que quieras estudiar. Y allá no hay muchas. Hay facultades pero que por ahí no hay muchas para elegir. Entonces por ese motivo no volvería, por mis hijos (...)

M: ¿Vos no te imaginás volviendo a Bolivia en algún momento?

$\mathrm{E}:$ Sí, mi plan es ponle que ahora encaminarlos a mis hijos, ponle, hasta que tengan su profesión, decidan qué es lo que quieran estudiar, esto y lo otro, yo creo que a la larga yo me voy a ir, irme e ir a morir a mis tierras, como dicen. Porque yo a veces digo, yo les voy acompañar hasta donde terminen su carrera y ahí decidirán qué es lo que quieren ustedes. Y yo hasta ahí los voy a acompañar. Y después volverme (Ester).

Los migrantes entrevistados llegan a Argentina por motivos económicos o de vulneración personal. No obstante, una vez aquí añoran algunas características de sus países natales, como la presencia de sus familiares, vivir en zonas más tranquilas y seguras. Frente a ello, uno de los motivos centrales por los cuales continúan viviendo 
REVISTA DE LA ESCUELA DE CIENCIAS DE LA EdUCACIÓN, AÑO 16, NRO. 15, VOL. 1, ENERO A JUNIO DE 2020. PÁGINAS 21-33. ISSN 2362-3349 (EN LÍNEA). ENTRE EL AFECTO, LA AUTORIDAD Y LA TRANSMISIÓN: ANÁLISIS DE LOS VÍNCULOS INTERGENERACIONALES EN LAS EXPERIENCIAS ESCOLARES DE FAMILIAS MIGRANTES. MARIANA ALEJANDRA GONZÁLEZ.

en Argentina, es la educación de sus hijos ${ }^{4}$. Cuando comparan las escuelas de sus países, donde ellos estudiaron, y las de los niños a cargo en la actualidad, destacan de estas últimas la posibilidad de acceder a una carrera educativa, lo cual resulta difícil de alcanzar en sus ciudades de origen. A su vez, padres/madres se esfuerzan en apoyar a sus hijos en su trayectoria escolar. Se trata de un esfuerzo familiar, que cobra un sentido más profundo, como es el persistir viviendo en Buenos Aires para acompañar a los hijos un tiempo y luego, en un futuro, partir.

Por lo expuesto, se observa que los vínculos intergeneracionales entre padres e hijos se construyen a partir de un esfuerzo compartido. Se trata de una lógica de subjetivación, ya que implica un nuevo modo de conducirse, diferente al que ellos vivieron, signados por órdenes de mérito individual. Asimismo, también responde a la lógica de estrategia, en la medida en que los modos que encuentren para concretar sus metas, responden a balances racionales (Martuccelli, 2010).

\section{Consideraciones finales}

En este artículo se han recorrido diversos sentidos de los vínculos intergeneracionales (docentesestudiantes y padres-hijos) a partir de las experiencias escolares de los padres y los niños. A continuación, se presenta una síntesis de lo desarrollado en las secciones previas, donde puede apreciarse cómo se reconfiguran tales vínculos en un tiempo pasado y en la actualidad, en el marco de los procesos de mutación institucional.

Tabla 2. Vínculos intergeneracionales en relación padre-hijo y docente-alumno

\begin{tabular}{|c|c|}
\hline \multicolumn{2}{|c|}{ VINCULOS INTERGENERACIONALES } \\
\hline PADRE - HIJO & DOCENTE - ALUMNO \\
\hline Antes: relaciones más distantes & $\begin{array}{c}\text { Antes: modo disciplinar-autoritario. Tipo de } \\
\text { líder: tradicional y racional burocrático. } \\
\text { Convive con tratos afectuosos (escuela } \\
\text { como un soporte) }\end{array}$ \\
$\begin{array}{c}\text { Hoy: esfuerzos por construir vínculos } \\
\text { cercanos. Transmisión de propia historia } \\
\text { para que sus hijos eviten igual destino }\end{array}$ & $\begin{array}{c}\text { Hoy: líder de tipo carismático. Fragilidad de } \\
\text { la escuela como institución moderna, } \\
\text { conlleva a que los padres ejerzan }\end{array}$ \\
$\qquad \begin{array}{c}\text { Esfuerzo familiar en la dinámica diaria, a fin } \\
\text { de lograr un progreso social vía educación }\end{array}$ & $\begin{array}{c}\text { escolar. Persisten los tratos afectuosos. } \\
\text { ( }\end{array}$ \\
\hline
\end{tabular}

Fuente: Dato de trabajo de campo

En las experiencias escolares de los padres, se han destacado modos disciplinares y autoritarios por parte de los docentes, a la vez que espacios de contención y escucha. En las familias, los progenitores acatan las normativas escolares y se pronuncian de iguales maneras hacia los hijos. Los entrevistados vivían en sus hogares situaciones de vulneración de sus derechos, obligaciones domésticas o laborales y castigos físicos. Frente a lo cual,

\footnotetext{
${ }^{4}$ Desde los enfoques que estudian las movilidades se señalan el carácter dinámico de las mismas, de modo que los argumentos por los cuales se emigra o permanecen, no deben considerarse en términos absolutos, más bien plausibles de revertirse en cualquier momento.
} 
REVISTA DE LA ESCUELA DE CIENCIAS DE LA EDUCACIÓN, AÑO 16, NRO. 15, VOL. 1, ENERO A JUNIO DE 2020. PÁGINAS 21-33. ISSN 2362-3349 (EN LÍNEA). ENTRE EL AFECTO, LA AUTORIDAD Y LA TRANSMISIÓN: ANÁLISIS DE LOS VÍNCULOS INTERGENERACIONALES EN LAS EXPERIENCIAS ESCOLARES DE FAMILIAS MIGRANTES. MARIANA ALEJANDRA GONZÁLEZ.

la escuela deviene un soporte en sus biografías. Prima una lógica de integración, ya que los mandatos de la sociedad son los que comandan las experiencias sociales de los actores.

En las actuales trayectorias escolares de los niños, las docentes se dirigen hacia los alumnos de manera afectuosa, en este sentido continúa vigente el rol de soporte. Las prácticas autoritarias no se producen más, lo que es añorado por los padres, quienes consideran positiva la exigencia de respeto y disciplina. Esto genera una tensión, puesto que también valoran los tratos horizontales y la contención.

En los vínculos padre-hijo, los entrevistados manifiestan la búsqueda de relaciones cercanas con los niños, a diferencia de lo que ellos vivieron en su infancia. Aquí se destaca entonces una lógica de subjetivación, en la medida en que se construye un ser y hacer novedoso.

Estas relaciones tienen lugar en una transmisión de la propia experiencia. Los padres buscan narrar su historia, sus vivencias para que los hijos no repitan los errores que, en el ámbito escolar, sería que no abandonen los estudios. En parte, este ímpetu por la continuidad escolar se debe a que garantiza el ascenso social. Los actores consideran que, si los hijos estudian, en un futuro podrán desempeñarse en tareas laborales más calificadas, en comparación con la que ellos realizan.

Finalmente, los vínculos intergeneracionales suponen una cuota de esfuerzo familiar. Los padres se esfuerzan por acompañar a los hijos en sus trayectorias escolares. Esto supone una novedad (lógica de subjetivación) en comparación con aquellos logros y capacidades individuales que conllevaban al éxito escolar en sus infancias. Ahora se conducen de diversas maneras para que sus hijos no abandonen los estudios (lógica de estrategia).

Un elemento central en estas vinculaciones es el afecto, ya que las distancias entre adultos y niños, en ámbito familiar y escolar, están mediadas por relaciones amorosas. Por ello, resulta relevante, a futuro, interrogarse por los afectos, sensaciones, emociones como medios conductores de autoridad en las relaciones intergeneracionales.

\section{Referencias bibliográficas}

Araujo, K. (2016). El miedo a los subordinados: una teoría de la autoridad. Santiago: LOM.

Araujo, K. y Martuccelli, D. (2012). Desafíos comunes. Retrato de la sociedad chilena y sus individuos. Santiago de Chile: LOM.

Beheran, M. (2012). Migraciones y educación en Argentina. Transformaciones y continuidades. En S. Novick (dir) Migración y políticas públicas. Nuevos escenarios y desafíos. Buenos Aires: Catálogos.

Castro, J. y Regattieri, M. (org.) (2012). Interacción escuela-familia: insumos para las prácticas escolares. Brasilia: UNESCO

Cerletti, L. y Santillán, L. (2011). Familias y escuelas: repensando la relación desde el campo de la Antropología y la Educación. Boletín de Antropología y Educación, 2 (03), 7-16.

Cravino, C. (2009). Vivir en la villa. Relatos, trayectorias y estrategias habitacionales. Buenos Aires: UNGS.

Dubet, F. (2007). El declive y las mutaciones de la institución. Antropología Social, (16), pp.39-66.

Dubet, F. (2013). El trabajo de las sociedades. Buenos Aires: Amorrortu.

Dubet, F. y Martuccelli, D. (2000) ¿En qué sociedad vivimos? Buenos Aires: Losada.

Durkheim, E. (1984). Educación y sociología. México: Colofón.

Gallo, P. (2009). Transformaciones en las Relaciones Intergeneracionales, Autoridad y Violencias en las Escuelas. En: G. Noel (ed.) Violencia en las escuelas desde una perspectiva cualitativa. Buenos Aires: Ministerio de Educación.

González, M. (2018). Migración, escuela y familia. Un análisis de experiencias escolares de migrantes y sus hijos, en sectores populares de la Ciudad Autónoma de Buenos Aires. Tesis de Maestría, FLACSO (no publicada).

Honneth, A. (1997). La lucha por el reconocimiento. Por una gramática moral de los conflictos sociales. Barcelona: Crítica. Instituto Nacional de Estadística y Censos (INDEC), Censo Nacional de Población, Hogares y Viviendas, 2010.

Jarrín, A. (2014). Reseña: "Que no sean como nosotros": trayectoria formativo-laborales de los hijos de familias inmigrantes en el campo murciano, de A. Pedreño (coord.). Papeles del CEIC, (2), 1-7.

Juliano, D. (2002) Los desafíos de la migración. Antropología, educación e interculturalidad. Universidad de Barcelona. Anuario de Psicología, 33 (4), 487-498.

Ley de Educación Nacional, N²6.206 (2006).

Martuccelli, D. (2006). Lecciones de sociología del individuo. Pontificia Universidad Católica del Perú.

Martuccelli, D. (2010). Gramáticas del individuo. Buenos Aires: Losada.

Martuccelli, D. y De Singly, F. (2012). Las sociologías del individuo. Santiago: LOM.

Nicastro, S. y Greco, M. (2009). Entre trayectorias. Escenas y pensamientos en espacios de formación. Rosario: Homo Sapiens.

Novaro, G.; Padawer, A. y Hecht, A. (2015) (coord.) Educación, pueblos indígenas y migrantes. Reflexiones desde México, Brasil, Bolivia, Argentina y España. Buenos Aires: Biblos.

Novick, S. (coord) (2015). Seminario permanente de migraciones. 20 años. Catálogos, UBA.

Rosseau, J. (1982). Emilio o de la educación. Buenos Aires: Centro Editor de América Latina.

Souza, M. (2009). La artesanía de la investigación cualitativa. Buenos Aires: Lugar. 
REVISTA DE LA ESCUELA DE CIENCIAS DE LA EDUCACIÓN, AÑO 16, NRO. 15, VOL. 1, ENERO A JUNIO DE 2020. PÁGINAS 21-33. ISSN 2362-3349 (EN LÍNEA). ENTRE EL AFECTO, LA AUTORIDAD Y LA TRANSMISIÓN: ANÁLISIS DE LOS VÍNCULOS INTERGENERACIONALES EN LAS EXPERIENCIAS ESCOLARES DE FAMILIAS MIGRANTES. MARIANA ALEJANDRA GONZÁLEZ

Strauss, A. y Corbin, J. (2006). Bases de la investigación cualitativa. Técnicas y procedimientos para desarrollar la teoría fundamentada. Bogotá: CONTUS.

Sustas, S. y Touris, C. (2013). Refugios afectivos: el amor en los nuevos tiempos. En P. Di Leo y A. Camarotti (ed.) "Quiero escribir mi historia". Vidas de jóvenes en barrios populares. Ciudad de Buenos Aires: Biblos.

Vaccotti, L. (2014). En los márgenes de la política. Migrantes y movilizaciones por el derecho a la vivienda en las villas de la ciudad de Buenos Aires. Tesis de doctorado. Facultad de Ciencias Sociales. UBA.

Vaccotti, L. (2017). Proceso migratorio y dinámica de la informalidad urbana en la Ciudad de Buenos Aires. Genealogía de un proceso sociológico. EURE, Estudios Urbano Regionales, 43 (129).

Weber, M. (1983). Economía y Sociedad. Buenos Aires: FCE.

\section{-ANEXO 1. Sistematización de muestra}

\begin{tabular}{|c|c|c|c|c|c|}
\hline $\begin{array}{l}\text { Pseudónimo } \\
\text { del/de la } \\
\text { entrevistado/a }\end{array}$ & País de origen & Edad & $\begin{array}{l}\text { Máximo nivel } \\
\text { escolar } \\
\text { alcanzado }\end{array}$ & Motivo abandono & Niños a cargo \\
\hline Mary & Paraguay & 32 & $2^{\circ}$ incompleto & Se escapa de la casa. & $\begin{array}{l}\text { Un hijo en primaria, } \\
\text { una hija en jardín } \\
\text { infantes. }\end{array}$ \\
\hline Ester & $\begin{array}{c}\text { Bolivia } \\
\text { (Cochabamba) }\end{array}$ & 41 & $\begin{array}{l}2^{\circ} \text { completo } \\
\text { (abandonó y } \\
\text { retomó luego) }\end{array}$ & $\begin{array}{l}\text { Se va de la casa. } \\
\text { Cuando su situación se } \\
\text { hace estable, retoma } \\
\text { estudios (en Bolivia). }\end{array}$ & $\begin{array}{l}\text { Hijos en primaria y } \\
\text { secundaria en } \\
\text { curso. }\end{array}$ \\
\hline Richard & Bolivia (Oruro) & 41 & $2^{\circ}$ incompleto & $\begin{array}{l}\text { "Por joven rebelde que } \\
\text { no supo aprovechar } \\
\text { oportunidades". }\end{array}$ & $\begin{array}{l}\text { Hijos en primaria y } \\
\text { secundaria en } \\
\text { curso. }\end{array}$ \\
\hline Lorelei & $\begin{array}{c}\text { Bolivia } \\
\text { (Cochabamba) }\end{array}$ & 33 & $1^{\circ}$ incompleto & $\begin{array}{c}\text { Ir a trabajar, situación } \\
\text { de violencia en la casa. } \\
\text { Escapa. }\end{array}$ & $\begin{array}{l}\text { Hijos en nivel } \\
\text { primario. }\end{array}$ \\
\hline Mario & Bolivia (La Paz) & 48 & $2^{\circ}$ incompleto & $\begin{array}{c}\text { Fallece el padre, sale a } \\
\text { trabajar junto con sus } \\
\text { hermanos. }\end{array}$ & $\begin{array}{l}\text { Hija en primaria en } \\
\text { curso, hijos en } \\
\text { secundaria (en } \\
\text { curso y abandono). }\end{array}$ \\
\hline Dominique & $\begin{array}{c}\text { Bolivia } \\
\text { (Cochabamba) }\end{array}$ & 56 & $3^{\circ}$ incompleto & $\begin{array}{l}2^{\circ} \text { termina. Quiere } \\
\text { hacer } 3^{\circ} \text { de adultos. } \\
\text { Arranca, pero hay } \\
\text { compatibilidad con } \\
\text { tareas domésticas. }\end{array}$ & $\begin{array}{l}\text { Hijos mayores de } 18 \\
\text { años (abandono, } \\
\text { uno retomó con plan } \\
\text { Fines, otro en } \\
\text { curso). Nietos a } \\
\text { cargo en } 1^{\circ} \text {. }\end{array}$ \\
\hline
\end{tabular}


REVISTA DE LA ESCUELA DE CIENCIAS DE LA EDUCACIÓN, AÑO 16, NRO. 15, VOL. 1, ENERO A JUNIO DE 2020. PÁGINAS 21-33. ISSN 2362-3349 (EN LÍNEA). ENTRE EL AFECTO, LA AUTORIDAD Y LA TRANSMISIÓN: ANÁLISIS DE LOS VÍNCULOS INTERGENERACIONALES EN LAS EXPERIENCIAS ESCOLARES DE FAMILIAS MIGRANTES. MARIANA ALEJANDRA GONZÁLEZ.

\begin{tabular}{|c|c|c|c|c|c|}
\hline Roberta & $\begin{array}{c}\text { Paraguay } \\
\text { (Eusebio Ayala) }\end{array}$ & 60 & $\begin{array}{c}1^{\circ} \text { completo } \\
\text { (Abandonó en } \\
\text { Paraguay. } \\
\text { Retomó en Bs } \\
\text { As. Completó } 1^{\circ} \\
\text { en 2016) }\end{array}$ & $\begin{array}{c}\text { No hubo incentivo de } \\
\text { su familia. Las mujeres } \\
\text { destinadas al ámbito } \\
\text { doméstico. }\end{array}$ & $\begin{array}{c}\text { Hijos mayores de 18 } \\
\text { años en } 2^{\circ} \\
\text { abandono. Nietos y } \\
\text { sobrina a cargo, en } \\
1^{\circ} \text { en curso. }\end{array}$ \\
\hline
\end{tabular}

Fuente: Datos de trabajo de campo. 